\title{
Werner Sengenberger
}

\section{Arbeitsmarkt, Beschäftigung und Einkommen in Entwicklungsländern im Kontext wirtschaftlicher Globalisierung}

\begin{abstract}
Entgegen den Verheißungen der Globalisierungstheorie ist es in den vergangenen Jahrzehnten im Weltmaßstab nicht zu einer Angleichung der Beschäftigungs- und Lebensverhältnisse gekommen. Soziale Ungleichheit ist zwischen und innerhalb der Nationalstaaten gewachsen. Lediglich die Regionen in Ost- und Südostasien konnten durch Handel und Kapitalzuflüsse hohes Wirtschaftswachstum erzielen, die Armut reduzieren und gegenüber den Industriestaaten aufholen, allerdings um den Preis verstärkter Einkommensungleichheit innerhalb der Länder. Die große Mehrzahl der Entwicklungsländer konnte sich ökonomisch wenig oder nicht verbessern. Arbeitslosigkeit, informelle und prekäre Beschäftigung, sowie Armut verblieben auf hohem Niveau oder wuchsen teilweise noch an.
\end{abstract}

\section{$1 \quad$ Einleitung}

In Laufe der vergangenen drei Jahrzehnte haben sich Wirtschaft, Arbeitsmarkt, Arbeitsformen und Lebensstandard in den Entwicklungsländern unter dem Einfluss der Globalisierung grundlegend gewandelt. Nahezu alle Länder des Südens öffneten ihre Märkte, um sich in die Weltwirtschaft zu integrieren. Die Öffnung erfolgte teils freiwillig, teils erzwungenermaßen infolge der Konditionen der Kreditvergabe seitens der internationalen Finanzinstitutionen und anderer Gläubiger und einer aggressiven Exportpolitik der reichen Staaten im Rahmen multi- und bilateraler Handelsabkommen. Die wirtschaftlichen und sozialen Wirkungen der Marktöffnung waren infolge unterschiedlicher nationaler Politik der Entwicklungsländer höchst ungleich. In allen Ländern jedoch zeigte sich eine erhebliche Zunahme sozialer Ungleichheit.

Der Beitrag dokumentiert zunächst die Beschäftigungs-, Arbeitsmarkt- und Einkommensentwicklung mit Hilfe der wichtigsten wirtschafts- und sozialstatistischen Indikatoren. Es folgt eine Analyse des Einflusses der Globalisierungsinstrumente, vor allem der Handels- und Kapitalströme, sowie der dahinter stehenden Politik der Marktliberalisierung. Es wird aufgezeigt, wieweit das der Globalisierung unterliegende Freihandelsdogma tatsächlich praktiziert wurde und welchen Nutzen und Schaden dieses für die Entwicklung gestiftet hat. 


\section{Trends von Wachstum, Beschäftigung und Arbeitsmarkt in Entwicklungsländern}

Der weit überwiegende Teil der Weltbevölkerung insgesamt wie auch der Erwerbsbevölkerung lebt in Entwicklungsländern. Seit geraumer Zeit vollzieht sich eine Umschichtung des globalen Arbeitskräftepotenzials hin zu diesen Ländern. Dieses nimmt jährlich um rd. 50 Mio. Menschen zu. 97\% dieser Zunahme erfolgt in Entwicklungsländern. Im Jahr 2010 werden schätzungsweise 57\% aller Arbeitskräfte in Asien leben - ein Viertel allein in China. Auch in den übrigen Entwicklungsregionen, d. h. Afrika südlich der Sahara (=ASS), Naher Osten und Nordafrika sowie Lateinamerika und Karibik, wächst der Anteil am weltweiten Arbeitskräftepotenzial, während es in den Industriestaaten proportional auf 13,6\% und in den Reformstaaten (im Übergang von der Plan- zur Marktwirtschaft) auf 6,4\% sinkt (ILO 2003a, 5).

Tab. 1: Arbeitsmarkt- und wirtschaftliche Indikatoren nach Weltregionen, 1993 und 2003

\begin{tabular}{|c|c|c|c|c|c|c|c|}
\hline \multirow{2}{*}{$\begin{array}{r}\text { Region } \\
\text { Jahr }\end{array}$} & \multicolumn{2}{|c|}{$\begin{array}{l}\text { Beschäfti- } \\
\text { gungsquote }\end{array}$} & \multicolumn{2}{|c|}{$\begin{array}{l}\text { Arbeitslosen- } \\
\text { quote }\end{array}$} & \multirow{2}{*}{$\begin{array}{c}\text { Arbeitspro- } \\
\text { duktivität } \\
\text { \% pro Jahr } \\
\mathbf{1 9 9 3 - 2 0 0 3}\end{array}$} & \multirow{2}{*}{$\begin{array}{c}\text { Arbeitskräfte } \\
\text { potenzial } \\
\text { \% pro Jahr }\end{array}$} & \multirow{2}{*}{$\begin{array}{c}\text { BIP- } \\
\text { Wachstum } \\
\% \text { pro Jahr } \\
\mathbf{1 9 9 3 - 2 0 0 3}\end{array}$} \\
\hline & 1993 & 2003 & 1993 & 2003 & & & \\
\hline Alle Regionen & 63,3 & 62,5 & 5,6 & 6,2 & 1,0 & 1,8 & 3,5 \\
\hline $\begin{array}{l}\text { Lateinamerika u. } \\
\text { Karibik }\end{array}$ & 59,3 & 59,3 & 6,9 & 9,8 & 0,1 & 2,3 & 2,6 \\
\hline Ostasien & 78,1 & 76,6 & 2,4 & 3,3 & 5,8 & 1,3 & 8,3 \\
\hline Südostasien & 68,0 & 67,1 & 3,9 & 6,3 & 2,0 & 2,4 & 4,4 \\
\hline Südasien & 57,0 & 57,0 & 4,8 & 4,8 & 3,3 & 2,3 & 5,5 \\
\hline $\begin{array}{l}\text { Naher Osten \& } \\
\text { Nordafrika }\end{array}$ & 45,4 & 46,4 & 12,1 & 12,2 & 0,1 & 3,3 & 3,5 \\
\hline $\begin{array}{l}\text { Afrika südlich der } \\
\text { Sahara }\end{array}$ & 65,6 & 66,0 & 11,0 & 10,9 & $-0,2$ & 2,8 & 2,9 \\
\hline Reformstaaten & 58,8 & 53,5 & 6,3 & 9,2 & 2,3 & $-0,1$ & 0,2 \\
\hline Industriestaaten & 55,4 & 56,1 & 8,8 & 6,8 & 1,4 & 0,8 & 2,5 \\
\hline
\end{tabular}

Quelle: ILO World Employment Report 2004-05, 27

Die Beschäftigungsquote, d.h. der Anteil der Beschäftigten an der Bevölkerung im Erwerbsalter (ab 15 Jahren), ist im Weltmaßstab von 1993 bis 2003 geringfügig (von 63,3 auf 62,5\%) gesunken (vgl. Tabelle 1). Die Verringerung geht vor allem auf das Konto der Beschäftigungsminderung in Ost- und Südostasien - bedingt durch eine verbesserte Einkommenssituation - und in den Reformstaaten - bedingt durch starke Produktionseinbrüche nach dem Systemwechsel. In den übrigen Regionen blieb die Quote im Durchschnitt konstant oder erhöhte 
sich - darunter auch in den entwickelten Industrieländern (ILO 2005a, Tabelle 1.3,27). Die Zahlen widersprechen der These eines generellen Rückgangs der Erwerbsarbeit.

Wie Tabelle 2 zeigt, streut die Beschäftigungsquote international sehr stark - und zwar relativ unabhängig vom Entwicklungsstand oder mittleren Einkommensniveau eines Landes. Die höchsten Quoten von über 80\% werden in Nepal und einigen afrikanischen Ländern erreicht, Quoten von über 75\% erzielen China und skandinavische Länder. Die Quoten der Frauen differenzieren stärker als die der Männer. Dies erklärt sich vor allem durch die geringe Erwerbstätigkeit von Frauen in vorwiegend katholischen und vor allem islamischen Ländern. In den letzteren liegt die Quote zum Teil unter 10\%. Die höchsten Anteile beschäftigter Frauen finden sich wiederum in den ärmsten Ländern und im egalitär orientierten Norden Europas.

Die Arbeitslosigkeit ist global gesehen seit den 1960er Jahren stark angestiegen. Der Anstieg ist in erster Linie die Folge der Verlangsamung des wirtschaftlichen Wachstums. Das reale Bruttoinlandsprodukt sank weltweit gesehen von 5,3\% in den 1960er Jahren auf 3,5\% in den 1970er, 3,2\% in den 1980er und 2,5\% in den 1990er Jahren. Allein von 1990 bis 2004 erhöhte sich nach ILO-Angaben die Zahl der Erwerbslosen weltweit von rd. 100 Mio. auf 192 Mio. Die Entwicklungsländer waren höchst unterschiedlich betroffen: Die Arbeitslosenquote stieg in Lateinamerika und der Karibik und - auf relativ niedrigem Niveau - in Ost- und Südostasien. Sie blieb konstant auf relativ niedrigem Niveau in Südasien und auf hohem Niveau in Afrika und im Nahen Osten. Die Quote erhöhte sich im Durchschnitt aller Reformstaaten und fiel in den Industrieländern (vgl. Tabelle 1).

Aus mehreren Gründen ist die Arbeitslosenquote in Entwicklungsländern als Indikator der Beschäftigungslage allerdings wenig brauchbar: Sie wird zumeist nur in den städtischen Ballungsräumen und für bestimmte Wirtschaftssektoren gemessen; sie bezieht sich nur auf die unselbstständig Beschäftigten, die in vielen Ländern nur eine Minderheit unter den Erwerbstätigen bilden, während die Selbstständigen allein oder zusammen mit den mithelfenden Familienangehörigen in der Mehrheit sind. Der Anteil der Selbstständigen an den Beschäftigten ausserhalb der Landwirtschaft beträgt in Sub-Sahara Afrika im Durchschnitt 53\%, in der Karibik 55\%, in Südamerika 43\% und in Südasien 50\%. In den „Arbeitsgesellschaften” Europas und Nordamerikas bewegt sich der Selbstständigenanteil dagegen nur um die 10\% (Tabelle 2). Darüber hinaus gibt es in vielen Entwicklungsländern keinen oder nur einen begrenzten institutionellen Anreiz, die Arbeitslosigkeit anzuzeigen: Wo sollen sich Arbeitslose melden, wenn es keine Arbeitsvermittlung gibt oder diese geografisch nicht erreichbar ist? Selbst wenn es eine Arbeitsverwaltung gäbe, wäre es für den Arbeitslosen wenig interessant, sich dort einzufinden, insoweit keine Lohnersatzleistungen bei Arbeitslosigkeit gezahlt werden, (was nach Schätzung der ILO für rd. 75\% aller Arbeitslosen in Entwicklungsländern der Fall ist). Gehört man nicht zu den Eliten, kann man es sich gar nicht leisten, arbeitslos zu sein. Viele Länder betreiben auch keine aktive Arbeitsmarktpolitik, die den Arbeitssuchenden helfen könnte, ihre Beschäftigungsfähigkeit zu verbessern und eine neue Stelle zu finden.

Besser als durch die Arbeitslosenquote werden Beschäftigungsdefizite in Entwicklungsländern durch den Indikator „Unterbeschäftigung” erfasst. Nach einer ILO-Resolution von 1998 ist eine Arbeitskraft unterbeschäftigt, wenn sie weniger Stunden arbeitet als sie möchte und fähig ist. Die Größenordnung für diese sog. zeitbezogene Unterbeschäftigung beträgt in armen Ländern zumeist ein Mehrfaches der Werte für die reichen Staaten (vgl. dazu ILO Schlüsselindikator 12, ILO 2003b, 395f). Auch dieser Indikator trägt jedoch nur begrenzt zur Erhellung der Beschäftigungssituation bei, denn in vielen Entwicklungsländern sind die Arbeitszeiten extrem lang, so dass typischerweise zeit- und mengenmäßige Unter- und 
Tab. 2: Beschäftigungsquote und Beschäftigungsstatus für ausgewählte Länder in 2001

\begin{tabular}{|c|c|c|c|c|c|c|c|c|}
\hline \multirow[t]{2}{*}{ Land } & \multirow{2}{*}{$\begin{array}{l}\text { Entwick- } \\
\text { lungsstand }\end{array}$} & \multirow{2}{*}{$\begin{array}{l}\text { Einkom- } \\
\text { mensniveau }\end{array}$} & \multicolumn{3}{|c|}{ Beschäftigungsquote } & \multicolumn{3}{|c|}{ Beschäftigtenstatus } \\
\hline & & & $M+F$ & $M$ & $F$ & Abhäng & Selbstständ. & Mithelf. \\
\hline Dänemark & hoch & hoch & 76,9 & 81,5 & 72,2 & 91,2 & 8,8 & \\
\hline Schweden & hoch & hoch & 76,5 & 78,6 & 74,3 & 90,0 & 9,7 & 0,3 \\
\hline Frankreich & hoch & hoch & 49,9 & 57,4 & 43,1 & 91,1 & 8,9 & - \\
\hline Deutschland & hoch & hoch & 52,9 & 61,4 & 44,8 & 88,9 & 9,9 & 1,2 \\
\hline Italien & hoch & hoch & 44,1 & 57,4 & 31,7 & 72,3 & 23,6 & 4,2 \\
\hline USA & hoch & hoch & 63,8 & 70,8 & 57,3 & 92,6 & 7,3 & 0,1 \\
\hline Japan & hoch & hoch & 58,9 & 71,1 & 48,6 & 83,7 & 10,8 & 5,1 \\
\hline$\overline{\text { Singapur }}$ & hoch & hoch & 65,5 & 77,8 & 52,7 & 86,1 & 12,9 & 0,9 \\
\hline Tschechien. & mittel & mittel & 55,1 & 64,7 & 46,2 & 84,0 & 15,4 & 0,6 \\
\hline Polen & mittel & mittel & 46,1 & 53,0 & 39,7 & 72,0 & 22,8 & 5,5 \\
\hline Russland & mittel & mittel & 51,0 & 58,0 & 45,1 & 92,6 & 7,2 & 0,2 \\
\hline Marokko & mittel & mittel & 22,1 & 34,7 & 9,9 & 72,9 & 18,4 & - \\
\hline Algerien & mittel & mittel & 28,2 & 48,8 & 7,2 & & & \\
\hline Ägypten & mittel & mittel & 45,2 & 72,3 & 17,2 & 59,9 & 28,5 & 11,5 \\
\hline Südafrika & mittel & mittel & 39,4 & 46,4 & 33,1 & 80,7 & 18,2 & 1,0 \\
\hline Ghana & mittel & niedrig & 80,8 & 80,8 & 80,5 & 16,9 & 81,2 & - \\
\hline China & mittel & mittel & 75,6 & - & - & - & - & - \\
\hline Rep.Korea & mittel & mittel & 58,6 & 70,5 & 47,3 & 62,4 & 28,5 & 9,1 \\
\hline Bangladesh & mitttel & niedrig & 69,7 & 84,4 & 54,0 & 12,6 & 35,3 & 33,8 \\
\hline Nepal & mittel & niedrig & 84,3 & 88,3 & 80,5 & - & - & - \\
\hline Thailand & mittel & mittel & 67,7 & 75,2 & 60,5 & 39,6 & 33,5 & 29,9 \\
\hline Vietnam & mittel & niedrig & 74,3 & - & - & & & \\
\hline Mexiko & mittel & mittel & 57,6 & 80,5 & 37,1 & 62,7 & 28,6 & 8,7 \\
\hline Brasilien & mittel & mittel & 54,8 & 67,4 & 43,1 & 56,4 & 20,5 & - \\
\hline Chile & mittel & mittel & 49,1 & 67,0 & 31,9 & 68,6 & 29,4 & - \\
\hline Pakistan & niedrig & niedrig & 39,5 & 66,1 & 11,3 & 35,6 & 43,0 & 21,4 \\
\hline Jemen & niedrig & niedrig & 28,2 & 52,1 & 4,2 & - & - & - \\
\hline Tanzania & niedrig & niedrig & 82,0 & 85,0 & 79,2 & - & - & - \\
\hline Uganda & niedrig & niedrig & 59,9 & 58,7 & 60,9 & 13,7 & 50,8 & - \\
\hline Äthiopien & niedrig & niedrig & 69,4 & 80,1 & 58,5 & 8,8 & 51,5 & - \\
\hline
\end{tabular}

Quellen: KILM, 3rd edition, 2004; *UNDP, HDR; ** Weltbank, World Development Indicators 
Überbeschäftigung koexistieren. Man weilt täglich und wöchentlich lange an einem oder mehreren Arbeitsplätzen, ist aber nicht kontinuierlich produktiv tätig und in diesem Sinne nicht vollbeschäftigt (vor allem in den ärmeren Entwicklungsländern sind vielfach Arbeitsplatz und familiärer Haushalt räumlich nicht voneinander getrennt). Da infolge des diskontinuierlichen Arbeitsflusses - und auch wegen des typischerweise geringen Kapitaleinsatzes - die Arbeitsproduktivität niedrig ist, muss man versuchen, viele Stunden einzubringen, um am Ende ein existenzsicherndes Einkommen zu erzielen.

Von zentraler Bedeutung für die Kennzeichnung der Beschäftigungssituation in Entwicklungsländern ist der Anstieg der „,informellen Wirtschaft” und der „informellen Beschäftigung” in den vergangenen drei Jahrzehnten. Hierbei handelt es sich um Unternehmen und Arbeitskräfte, die außerhalb des geltenden rechtlichen Rahmens tätig sind. Es sind Betriebe und Selbstständige, die nicht amtlich registriert sind und keine Steuern und Abgaben entrichten; und um Arbeitskräfte, die als Unselbstständige in weitgehend oder vollends illegalen, ungeregelten und ungeschützten Arbeitsverhältnissen stehen und z. T. hochprekären Arbeitsbedingungen ausgesetzt sind. Im Gegensatz zu den entwickelten Ländern, in denen informelle Beschäftigung allgemein eher im Verborgenen erfolgt oder wegen Illegalität absichtlich verheimlicht wird, tritt sie in den Entwicklungsländer größtenteils sehr sichtbar zu Tage, insoweit sie im Freien, zumeist auf der Straße und auf öffentlichen Plätzen ausgeführt wird. Üblicherweise werden informelle Klein- und Kleinstbetriebe, selbst wenn sie illegal sind, von den Behörden geduldet. Oft machen die Betriebe durch Werbung und Beschilderung auf sich aufmerksam, um Kunden zu gewinnen (Daza 2005, 13).

Einigermaßen verläßliche Daten über informelle Beschäftigung liegen für 47 Entwicklungsländer vor. Unter diesen Betrug der Anteil der informell Beschäftigten in städtischen Räumen in 17 Ländern mehr als 50\%. Darunter waren Peru (54\%), Mali (71\%), Äthiopien (51\%), Tanzania (67\%), Indien (56\%), Pakistan (65\%) und Nepal (73\%). Die Quoten der Frauen in der informellen Beschäftigung lagen teilweise noch darüber. In Ländern, für die Zeitreihen zur informellen Beschäftigung verfügbar sind, zeigt sich ein deutlicher Anstieg der Quoten, ausgenommen Staaten in Ost- und Südostasien, wo informelle Aktivitäten stark zurückgegangen sind. Besonders krass ist die Zunahme der informellen Wirtschaft in einer Reihe von GUS-Staaten (ILO 2002a, 12f). Ein hoher Prozentsatz der von Arbeitsarmut betroffenen Arbeitskräfte ist in der informellen Wirtschaft tätig. ${ }^{1}$

Die Aktivitäten in der informellen Wirtschaft, die in vielen Ländern nahezu alle Wirtschaftbereiche überspannen - weshalb man auch den vormaligen Begriff „informeller Sektor” durch den Begriff „informelle Wirtschaft” ersetzt hat - sind sehr vielfältig. Neben auf das pure Überleben gerichtete, traditionelle Tätigkeiten, wie Reparaturen von Werkzeugen und Geräten, Schuhputzen, Straßenverkauf und Müllsammeln und -verwertung erwächst die rapide Zunahme der informellen Wirtschaft in vielen Ländern aus der Umwandlung formeller Unternehmen zwecks Steuerersparnis in informelle; oder der gezielten Auslagerung von Produktion in informelle Betriebe oder an frühere Mitarbeiter, die jetzt auf eigene Rechnung wirtschaften - häufig geduldet oder sogar unterstützt durch staatliche Stellen zur Verringerung von Lohnkosten und Soziallasten und zum (vermeintlichen) Gewinn von Attraktivität des Standorts für ausländische Investoren (Portes 1994, 165f). Die in den 1970er Jahren von der ILO und anderen gehegte Erwartung, dass die informelle Wirtschaft zu einer marginalen

1 Die weltumspannende Betrachtung und statistische Analyse großer Regionsblöcke in diesem Beitrag bringt es mit sich, dass differenzierte, mitunter sogar gegenläufige Enwicklungen in einzelnen Ländern nicht immer ausreichend gewürdigt werden können. Allerdings sind Abweichungen von regionalen Trends auch nicht übermäßig zahlreich. 
Größe wird, da sie als nachholende Imitation des Industrialisierungsprozesses der westlichen kapitalistischen Ländern sukzessive von der formellen Wirtschaft absorbiert wird, hat sich nicht bewahrheitet (zu einer Neuinterpretion des informellen Sektors in Lateinamerika ,vgl. Pries 1992, 665f; zur informellen Wirtschaft in Indien vgl. Sivananthiram/Venkata Ratnam 2005).

Ein weiteres, herausragendes Merkmal von Arbeit in den ärmeren Entwicklungsländern ist die durchschnittlich niedrige Kapitalproduktivität und die geringe Arbeitsproduktivität, gemessen als Output pro Erwerbstätigen, durch verbreitet unproduktive Tätigkeiten, die teilweise mit der Informalisierung von Beschäftigung einhergehen. Der Zusammenhang zwischen niedriger Arbeitsproduktivität, niedrigen Arbeitseinkommen und Armut ist empirisch vielfach nachgewiesen (ILO 2005a, 95f). Die Entwicklung der Arbeitsproduktivität divergiert aber wiederum ausserordentlich stark zwischen den Ländergruppen und einzelnen Ländern, sowie auch innerhalb der Länder. Sie konnte seit 1993 in Ostasien stark gesteigert werden - im Mittel um jährlich 5,8\%. In Südasien lag der Zuwachs bei 3,3\%, in Lateinamerika blieb das Wachstum nahezu aus (0,1\%) und in ASS war er negativ (-0,2\%). Die enormen Differenzen im durchschnittlichen jährlichen Zuwachs der Arbeitsproduktivität zeigen sich für den Zeitraum 1980-2000 auch in den folgenden Werten einzelner Länder: Republik Korea (5,6\%), Sri Lanka (5,5\%), China (5,0\%), Singapur (4,3\%), Thailand (4,1\%), Indien $(3,4 \%)$, Venezuela $(0,0 \%)$, Brasilien (-0,2\%), Philippinen (-0,9\%), Mexiko (-1,5\%), Peru $(-1,6)$ (ILO, 2005a, 4).

Arbeitslosigkeit, Unterbeschäftigung, informelle Beschäftigung und niedrige Produktivität kumulieren in einem hohen Volumen von Einkommensarmut. Viele Entwicklungsländer haben in den vergangenen zwei Jahrzehnten extreme wirtschaftliche und finanzielle Krisen erlebt und - wie Mexiko, Nicaragua, Brasilien, Argentinien und Ghana - Phasen von Hyperinflation durchgemacht. Dies hat tiefe Spuren hinterlassen. Des Weiteren muss darauf hingewiesen werden, dass Armut auch andere wichtige Ursachen hat. Zu nennen sind Bürgerkrieg und damit zusammenhängende, übersteigerte Rüstungs- und Militärausgaben, Genozid, Naturkatastrophen, HIV/AIDS und andere Epi- und Pandemien, unsauberes Trinkwasser, ein allgemein schlechter Gesundheitszustand der Erwerbsbevölkerung durch Unteroder Mangelernährung, Bodenerosion, hohe Preise für Düngemittel, usf. Die verschiedenen Ursachen sind allerdings nicht unabhängig voneinander, sondern stehen zumindest teilweise in Wechselwirkung zueinander.

Bei der Vermessung von Einkommensarmut ist zu unterscheiden zwischen „,absoluter” oder „extremer” Armut und „relativer” Armut. Bei Ersterer wird ein bestimmtes Mindesteinkommen zugrunde gelegt, bei Letzterer ein bestimmter Prozentsatz - zumeist ein Drittel - des Durchschnittseinkommens. Global gesehen ist die Quote der Armen zwischen 1980 und 2003 von 39,7\% auf 19,5\% gesunken, wenn man von einem Einkommen von 1,08 US \$ pro Kopf und pro Tag ausgeht (gemessen in Preisen von 1993 und internationaler Kaufkraftparität). Konsum im Wert von 1 US \$ (eingeschlossen Naturallohn und Eigenproduktion), ist nach Einschätzung der Weltbank das absolute Minimum dessen, was in Entwicklungsländern zum blanken Überleben erforderlich ist. Mäßiger war der Rückgang der Armut auf der Basis von 2 US \$ pro Tag - nämlich von 65,7\% auf 51,2\%. Eine Verringerung der Armutsrate ergab sich vor allem in Ostasien und Südasien. Die Armut veränderte sich durchschnittlich wenig in Lateinamerika und der Karibik und stieg in ASS von einem bereits hohen Niveau weiter an (ILO 2005a, 23). Die bislang optimistischen Prognosen der Weltbank für eine weitere Senkung der Armutsqoten bis 2015 wurden mittlerweile stark nach unten korrigiert (World Bank Daily Press, 12.12.2005). Betrachtet man anstelle der Quoten die Zahl der Armen, so 
zeigt sich auf der Basis der 1 \$-Schwelle im Zeitraum 1981-2001 eine globale Erhöhung der Armut bei der außerhalb Chinas lebenden Weltbevölkerung. In Lateinamerika war die Zunahme mäßig, in Afrika hat sie sich fast verdoppelt (Chen/Ravallion 2005, 16).

Tab. 3: Anteile (in \%) der von Armut betroffenen Erwerbsbevölkerung, nach Region und ausgewählten Jahren

\begin{tabular}{|l|l|l|l|l|l|l|l|l|l|l|}
\hline Region & \multicolumn{5}{|c|}{$\begin{array}{c}\text { Leben von weniger als } \\
\text { 1 US \$ pro Tag }\end{array}$} & \multicolumn{5}{c|}{ Leben von weniger als } \\
& & \multicolumn{4}{|c|}{ US \$ pro Tag } \\
\hline & & 1980 & 1990 & $2003^{*}$ & $2015^{* *}$ & & 1980 & 1990 & $2003^{*}$ & $2015^{* *}$ \\
\hline Alle Regionen & & 40,3 & 27,5 & 19,7 & 13,1 & & 59,8 & 57,2 & 49,7 & 40,8 \\
\hline $\begin{array}{l}\text { Lateinamerika u. } \\
\text { Karibik }\end{array}$ & 15,6 & 16,1 & 13,5 & 11,5 & & 41,2 & 39,3 & 33,1 & 28,8 \\
\hline Ost-Asien & 71,1 & 35,9 & 17,0 & 6,5 & & 92,0 & 79,1 & 49,2 & 25,8 \\
\hline Südost-Asien & & 37,6 & 19,9 & 11,3 & 7,3 & & 73,4 & 69,1 & 58,8 & 47,7 \\
\hline Südasien & 64,7 & 53,0 & 38,1 & 19,3 & & 95,5 & 93,1 & 87,5 & 77,4 \\
\hline $\begin{array}{l}\text { Naher Osten u. } \\
\text { Nahost }\end{array}$ & 5,0 & 3,9 & 2,9 & 2,3 & & 40,3 & 33,9 & 30,4 & 24,9 \\
\hline $\begin{array}{l}\text { Afrika südlich } \\
\text { der Sahara (ASS) }\end{array}$ & 23,4 & 55,8 & 55,8 & 54,0 & 85,5 & 89,1 & 89,0 & 87,6 \\
\hline Reformstaaten & 1,6 & 1,7 & 5,2 & 2,1 & & 1,7 & 5,0 & 23,6 & 9,8 \\
\hline
\end{tabular}

Quelle: Kapsos 2004, 12-22; * Schätzungen; ** Projektionen

Während Armut in den Industriestaaten vor allem die trifft, die nicht erwerbstätig sind, reicht sie in den Entwicklungsländern infolge verbreitet niedriger Arbeitsproduktivität und niedriger Arbeitseinkommen tief in die Erwerbsbevölkerung hinein. Die ILO ermittelt deshalb das Ausmaß von „Arbeitsarmut” (Kapsos 2004, 10). Danach verdienten im Jahr 2004 knapp 1,4 Mrd. Menschen, d. h. 48\% aller Arbeitskräfte weltweit, weniger als 2 US \$ pro Tag und 535 Mio. Menschen weniger als 1 US \$. Die Zahl der von Armut betroffenen Arbeitskräfte hat sich seit 1980 erhöht, legt man die Schwelle von 2 US \$ zugrunde. Auf Basis der 1 US \$ Marke war die Zahl dagegen rückläufig. Die Quote der Armen in der Erwerbsbevölkerung ist global gesehen beträchtlich gesunken, dies ist aber größtenteils auf den starken Rückgang der Armut im bevölkerungsreichen China und Indien zurückzuführen. Die übrigen Regionen verzeichneten entweder mäßig fallende, stagnierende oder erhöhte Quoten (Tabelle 3). Hinter den aggregierten globalen Zahlen verbirgt sich also ein stark regional differenziertes Bild der Armutsentwicklung.

Das Milleniumsziel Nr. 1 der Vereinten Nationen richtet sich auf die Halbierung der Quote der Armen und Hungernden im Zeitraum 1990 bis 2015. Tabelle 4 zeigt, dass auf der Basis der 1 US \$- Armutsschwelle und der Prognosen für das BIP-Wachstum dieses Ziel nur in Ost-, Südost- und Südasien erreichbar erscheint, nicht aber in den übrigen Regionen. Die Chance, die durch die 2 US \$- Schwelle definierte Armut in den nächsten zehn Jahren zu mindern, besteht lediglich in Ostasien. 
In vielen Entwicklungsländern funktioniert der klassische Reservearmeemechanismus, der in den Industrieländern durch das Arbeitsrecht und den Sozialstaat für einige Jahrzehnte erheblich abgeschwächt wurde, in großen Teilen der Wirtschaft nahezu uneingeschränkt.

\section{Tab. 4: Erforderliches BIP-Wachstum zur Halbierung der Arbeitsarmut bis 2015 und BIP-Wachstumsprognosen für den Zeitraum 1995-2005 (in \%)}

\begin{tabular}{|l|l|l|l|}
\hline Region & \multicolumn{2}{|c|}{$\begin{array}{c}\text { Erforderliches BIP-Wachstum um Arbeitsarmut } \\
\text { zu halbieren, auf Basis von }\end{array}$} & \multicolumn{1}{|c|}{$\begin{array}{c}\text { Wachstumsrate } \\
\text { 1995-2005 }\end{array}$} \\
\hline & \multicolumn{1}{|c|}{ US \$ pro Tag } & \multicolumn{1}{|c|}{ ( pro Tag } & \\
\hline $\begin{array}{l}\text { Alle Regionen außer Ost- } \\
\text { asien und Industriestaaten }\end{array}$ & 5,3 & über 10 & 3,8 \\
\hline Ostasien & $3-4$ & $6-8$ & 7,9 \\
\hline Südostasien & $4-5$ & über 10 & 4,1 \\
\hline Südasien & $5-6$ & über 10 & 5,8 \\
\hline Lateinamerika und Karibik & $3-4$ & $4-6$ & 2,4 \\
\hline $\begin{array}{l}\text { Naher Osten und Nord- } \\
\text { afrika }\end{array}$ & $4-5$ & $8-10$ & 4,0 \\
\hline Afrika südlich der Sahara & über 8 & über 10 & 3,7 \\
\hline Reformstaaten & $4-5$ & $8-10$ & 3,3 \\
\hline
\end{tabular}

Quelle: Kapsos 2004

Dazu tragen bei:

- fehlender oder schwacher Kündigungsschutz und/oder geringe Einkommensersatzleistungen im Falle von Arbeitslosigkeit, Krankheit, Invalidität und im Alter. Der Anteil der Arbeitskräfte, die Anspruch auf Leistungen in einem oder mehreren Systemen der sozialen Sicherung haben, wird von der ILO weltweit auf lediglich rd. 20\% geschätzt. In ASS und Südasien liegt der Prozentsatz bei nur rd. 10\%, in Lateinamerika zwischen 10\% und 80\% (van Ginneken 2003, 277). In Afrika gab es nach der Unabhängigkeit der Staaten vor 40 Jahren noch eine weitreichende soziale Sicherung, u. a. kostenlose staatliche Krankenversorgung.

- Fortbestand traditioneller Formen der Zwangsarbeit, Sklaverei und Schuldknechtschaft in einer beträchtlichen Zahl von Ländern und z.T erheblicher Anstieg neuer Formen von Zwangsarbeit, wie Handel von Frauen und Kindern und deren Beschäftigung in der Sexindustrie. Die Zahl der Zwangsarbeiter wird derzeit auf mindestens 12,3 Mio geschätzt (ILO 2005b, 10). Zwangsarbeit wird nach ILO-Konvention Nr. 29 (von 1930) definiert als „, unfreiwillige Arbeit oder Dienstleistung, bei deren Verweigerung die Arbeitskraft mit Bestrafung zu rechnen hat”. Die ILO-Konvention 105 von 1957 legt fest, dass Arbeitszwang zum Zwecke wirtschaftlicher Entwicklung, politischer Erziehung, Diskriminierung, Arbeitsdisziplinierung oder als Strafe für Beteiligung an Arbeitskämpfen unzulässig ist.

- Trotz einiger Erfolge bei der Bekämpfung der Kinderarbeit hat sich deren Umfang nach Schätzung der ILO allein von 2000 auf 2004 von 211 Mio. auf 246 Mio. ausgeweitet. Die größte Zahl regelmäßig arbeitender Kinder findet sich in Asien, der höchste Anteil 
an der Kinderbevölkerung in Sub-Sahara Afrika (ILO 2002, 19). Zwangsarbeit und Kinderarbeit führen zur Vergrößerung des Arbeitskräfteangebots und drücken damit die Löhne, womit der Arbeitszwang zur Überlebenssicherung weiter verstärkt wird.

- Verringerte Organisationsdichte und Verhandlungsmacht von Gewerkschaften in nahezu allen Entwicklungsländern infolge erhöhter Arbeitslosigkeit und wachsender informeller Beschäftigung, Privatisierung und anderer Folgen staatlicher Strukturanpassungspolitik, sowie Verweigerung der Vereinigungsfreiheit. Gewerkschafter wurden - insbesondere in Südostasien und Lateinamerika - vielfach politisch verfolgt, da sie als Regimekritiker auftraten und eine führende Rolle im politischen Widerstand spielten.

\section{$3 \quad$ Wachsende Einkommensungleichheit}

Die international stark divergierende Entwicklung der Arbeitsproduktivität spiegelt sich in wachsender Einkommensungleichheit der Regionen. Zwischen 1985 und 2002 ist das durchschnittliche BIP pro Kopf, in China und Indien um 240\% und in den Ländern mit hohen Einkommen um 180\% gewachsen. In Ländern mit mittlerem Einkommensniveau (ohne China) betrug die Steigerung lediglich 70\% und in den Ländern mit niedrigen Einkommen (Indien ausgenommen) nur 10\%. Entsprechend haben sich die Anteile dieser Ländergruppen am Weltsozialprodukt mächtig verschoben: Die Gruppe der ärmsten Länder ist von 4,5\% auf 2\% zurückgefallen, die Ländergruppe mit mittlerem Einkommen von 17,5\% auf 11,5\%. Die reichen Staaten hingegen konnten ihren Anteil von knapp drei Viertel auf mehr als vier Fünftel des Welteinkommens ausbauen, obgleich ihr Anteil an der Weltbevölkerung rückläufig war und jetzt nur noch $14 \%$ beträgt. China und Indien zusammen steigerten ihren Anteil von 4,4 auf 6\% (Gunter/van der Hoeven 2004, 10). Auch andere Studien belegen, dass sich das Gefälle der Einkommen zwischen den OECD-Ländern und China, Indien und anderen Staaten in Ost- und Südostasien vermindert, zwischen den OECD-Ländern und Sub-Sahara Afrika, Nordafrika und Naher Osten, Lateinamerika und Osteuropa indes ausgeweitet hat (Cornia 2003, 6).

China und Indien konnten ihre Anteile am weltweiten Exportvolumen und noch stärker am Zufluss ausländischen Kapitals - sowohl durch Direktinvestitionen wie Portfolioinvestitionen - in den vergangenen zwei Jahrzehnten vervielfachen. Die reichen Staaten konnten ihre sehr hohen Anteile nahezu halten (Gunter/van der Hoeven 2004,10). Die übrigen Regionen hingegen haben beträchtlich verloren. Bei den ausländischen Direktinvestitionen handelt es sich um die Gründung von ausländischen Niederlassungen oder Zweigbetrieben oder den langfristigen Erwerb von mindestens 10\% des Kapitals ausländischer Unternehmen. Portfolioinvestitionen werden als eher kurzfristige Finanzanlagen definiert, die kein dauerhaftes Interesse an einem ausländischen Unternehmen begründen.

Von den größeren Rohstofflieferanten abgesehen spielen die ärmsten Länder nur noch eine marginale Rolle im Welthandel und erhalten nahezu kein ausländisches Kapital. Hinzu kommt, dass sich neben der mengenmäßigen Umschichtung von Handels- und Kapitalströmen auch die Terms of Trade (Preisrelation von Exporten zu Importen) verschoben haben, und zwar wiederum zu ungunsten der armen Länder (vgl. UNCTAD Jahresberichte und Entwick- 
lungsindikatoren der Weltbank). Eine Gegenbewegung zeichnet sich jedoch neuerdings durch die Preissteigerungen bei Rohstoffen, insbesondere fossilen Energieträgern, ab. In seltenen Fällen allerdings profitieren von den erhöhten Erträgen die Armen.

Welche groteske Ausmaße das Einkommensgefälle zwischen reichen und armen Ländern angenommen hat, ist der Tabelle 5 zu entnehmen. Das auf der Basis von Kaufkraftparität berechnete pro-Kopf-Inlandsprodukt des reichsten Landes (Luxemburg) beträgt mehr als das Hundertfache desjenigen der ärmsten Staaten; die Einwohner der OECD-Staaten verfügen über durchschnittlich mehr als das Dreifache des Durchschnittseinkommens aller Staaten. Zwischen 1960 und 2002 ist in den 20 reichsten Ländern das Inlandsprodukt pro Kopf - diesmal in Marktpreisen gemessen - von 11.417 auf 32.339 US \$ (in konstanten Dollarwerten von 1995) angewachsen, in den 20 ärmsten Staaten indes lediglich von 212 auf 267 US \$ (Weltkommission zur sozialen Dimension der Globalisierung 2004, 37). In anderen Worten, die reichsten Länder sind sehr viel reicher geworden, die ärmsten Länder sind heute fast genau so arm wie vor 40 Jahren. Diese Entwicklung in den letzten vier Jahrzehnten beschleunigt den säkularen Trend zu wachsender internationaler Einkommensdisparität: Während im Jahr 1810 die Einkommensrelation zwischen den 20 reichsten und den 20 ärmsten Ländern noch 3:1 betrug, lag sie 1913 bereits bei 11:1 und wuchs weiter auf 35:1 in 1970 und 72:1 in 1992 (UNDP 1999, 38). Der Trend steht im krassen Widerspruch zur neo-liberalen Theorie der Globalisierung, die Konvergenz der Wirtschafts- und Lebensbedingungen verheißt.

Tabelle 5 zeigt für ausgewählte Länder auch Indikatoren binnennationaler Einkommensverteilung, einschliesslich Gini-Koeffizient (mit Werten von 0 bis 1), prozentuales Verhältnis der 10 reichsten zu den 10 ärmsten Prozent der Bevölkerung und Anteil der Bevölkerung mit einem Einkommen von weniger als 50\% des Medianeinkommens. Auch bei der Betrachtung dieser Messgrößen ergibt sich ein Bild von teilweise dramatisch hoher Ungleichheit zwischen Reichen und Armen, mit Spitzenwerten in lateinamerikanischen und westafrikanischen Ländern. Allerdings gibt es auch arme Länder, wie beispielsweise Nepal, Bangladesh und Tanzania mit geringer oder mäßiger Ungleichheit in der personellen Einkommensverteilung. Nach Aussage des Internationalen Währungsfonds ist im Durchschnitt der Länder, für die verlässliche Einkommensdaten vorliegen, der Gini-Index in den 1990er Jahren von durchschnittlich 0,40 auf 0,48 gestiegen. Besonders stark war der Anstieg in Russland und anderen GUS-Staaten. Eine Untersuchung der UN in 73 Ländern ergab, dass das Einkommensdifferenzial zwischen den Jahren 1960 und 2000 in 48 Ländern, die zusammen genommen rd. 80\% der Weltbevölkerung ausmachen, gewachsen ist, während es in 16 Ländern etwa unverändert blieb und in 9 Ländern sich verminderte (UNDP 2002, 20). Im letzten Jahrzehnt des 20. Jahrhunderts hat sich die Spreizung der Einkommen rapide verstärkt. Sie betrifft selbst Länder mit hohem Wirtschaftwachstum und rückläufigen Armutsquoten, wie China und Indien. Für China stellte die Weltbank fest, dass seit dem Beitritt des Landes zur Welthandelsorganisation (WTO) in 2001 fast 90\% der privaten Haushalte in den Städten Einkommensverbesserungen und höhere Konsumausgaben verzeichneten, während die Landbevölkerung im Durchschnitt 0,7\% an Einkommen einbüßte. Bei der ärmsten Bevölkerungsgruppe sank das Einkommensniveau sogar um 6\% (Agence France Presse, 21.02.2005). Einkommensverbesserungen im Durchschnitt bringen demnach nicht automatisch mehr Einkommensgleichheit mit sich.

Eine der wichtigen Teilkomponenten der Entwicklung der personellen Einkommensungleichheit sind wachsende Lohndifferenziale. In zwei Dritteln von 77 Ländern - darunter auch Entwicklungsländern -, in denen Zeitreihen zum interpersonellen Lohndifferenzial analysiert wurden, zeigte sich in den 1980er und 1990er Jahren ein deutlicher Anstieg, womit 
Tab. 5: Einkommensniveau, Einkommensungleichheit und Armut in ausgewählten Ländern, 2001

\begin{tabular}{|c|c|c|c|c|c|c|c|}
\hline \multirow[t]{2}{*}{ Land } & \multirow{2}{*}{$\begin{array}{l}\text { BIP per } \\
\text { capita } \\
\text { (PPP \$ in } \\
2001)\end{array}$} & \multicolumn{2}{|c|}{$\begin{array}{l}\text { Einkommensungleich- } \\
\text { heit }\end{array}$} & \multicolumn{3}{|c|}{ Armut } & \multirow{2}{*}{$\begin{array}{l}\begin{array}{l}\text { Arbeits- } \\
\text { armut }\end{array} \\
\text { Anteil } \\
\text { in } \%\end{array}$} \\
\hline & & Gini Index & $\begin{array}{l}\text { Reichste } \\
10 \% \text { zu } \\
\text { Ärmsten } \\
10 \%\end{array}$ & $\begin{array}{l}<50 \% \\
\text { vom } \\
\text { Median }\end{array}$ & $\begin{array}{l}<1 \$ \\
\text { proTag }\end{array}$ & $\begin{array}{l}<2 \$ \\
\text { proTag }\end{array}$ & \\
\hline & \multicolumn{7}{|c|}{ Länder mit hohem Durchschnittseinkommen } \\
\hline Luxemburg & 53.780 & 30,8 & 7,7 & 3,9 & & $0,3^{*}$ & \\
\hline USA & 34.320 & 40,8 & 16,6 & 17,0 & & $13,6^{*}$ & \\
\hline Dänemark & 29.000 & 24,7 & 8,1 & 9,2 & & & \\
\hline Deutschland & 25.350 & 38,2 & 14,2 & 7,5 & & $7,3^{*}$ & \\
\hline Schweden & 24.180 & 25,0 & 5,9 & 6,6 & & $6,3^{*}$ & \\
\hline Grossbritannien & 24.160 & 36,1 & 13,4 & 12,5 & & $15,7 *$ & \\
\hline \multicolumn{8}{|c|}{ Länder mit mittlerem Durchschnittseinkommen } \\
\hline $\begin{array}{l}\text { Tschechische } \\
\text { Republik }\end{array}$ & 14.720 & 25,4 & 5,2 & 4,9 & & & \\
\hline Südafrika & 11.290 & 59,3 & 65,1 & 11,5 & 35,8 & 12,6 & \\
\hline Polen & 9.450 & 31,6 & 7,8 & & & & \\
\hline Chile & 9.190 & 57,5 & 20,7 & 18,4 & 4,2 & & \\
\hline $\begin{array}{l}\text { Russische Föde- } \\
\text { ration }\end{array}$ & 7.100 & 48,7 & 20,3 & & 7,0 & 25,1 & \\
\hline Brasilien & 7.360 & 59,1 & 65,8 & & 9,9 & 25,3 & 5,1 \\
\hline Rumänien & 5.830 & 28,2 & 7,2 & & 2,8 & 27,5 & \\
\hline Peru & 4.570 & 46,2 & 22,3 & & 15,5 & 41,4 & 16,8 \\
\hline Ukraine & 4.350 & 29,0 & 6,4 & & 2,9 & 45,6 & \\
\hline China & 4.020 & 40,3 & 12,7 & & 18,5 & 53,7 & 19,1 \\
\hline Ägypten & 3.520 & 28,9 & 8,0 & & 3,1 & 52,7 & 3,3 \\
\hline \multicolumn{8}{|c|}{ Länder mit niedrigem Durchschnittseinkommen } \\
\hline Indonesien & 2.940 & 31,7 & 7,8 & & 7,7 & 55,3 & 15,7 \\
\hline Honduras & 2.830 & 59,0 & 49,1 & & 23,8 & 44,4 & 41,8 \\
\hline Indien & 2.840 & 37,9 & 9,5 & & 34,7 & 79,9 & 45,4 \\
\hline Bangladesh & 1.610 & 33,6 & 6,8 & & 29,1 & 77,8 & 30,0 \\
\hline Nepal & 1.310 & 36,7 & 9,3 & & 37,7 & 82,5 & 38,9 \\
\hline Kenia & 980 & 44,5 & 15,6 & & 26,5 & 62,3 & 27,3 \\
\hline Nigeria & 850 & 50,6 & 24,9 & & 70,2 & 90,8 & 72,4 \\
\hline Mali & 810 & 60,5 & 26,2 & & 72,8 & 90,5 & 75,1 \\
\hline Tanzania & 520 & 38,2 & 10,8 & & 19,9 & 59,7 & 20,5 \\
\hline Sierra Leone & 470 & 62,9 & 87,2 & & 57,0 & 74,5 & 56,1 \\
\hline OECD & 23.363 & & & & & & \\
\hline
\end{tabular}

Quelle: $\quad$ UNDP, Human Development Report 2003, ILO Global Employment Trends 2003,

*Anteil der Bevölkerung unter 11 US \$ pro Tag 
der verbreitete Trend zur Lohnnivellierung während der Jahre von 1950 bis 1970 umgekehrt wurde (Cornia 2003, 2). Verstärkt wurde die Einkommensdifferenzierung auch durch die Reformprogramme von IWF und Weltbank zur gesamtwirtschaftlichen Stabilisierung und Strukturanpassung der Entwicklungsländer (Cornia 2003, 20).

Arbeitslosigkeit, Unterbeschäftigung, Armut und das wachsende internationale Einkommensgefälle bilden den wirtschaftlichen Hintergrund für den Trend zu verstärkter intra- und interregionaler, teils legaler, teils illegaler Arbeitskräftewanderung, überwiegend von den weniger zu den mehr entwickelten Ländern. Die ILO schätzte die Zahl der Migranten und ihrer Familienangehörigen im Jahr 2000 auf etwa 120 Mio. Das waren 75 Mio. mehr als 1965. Zwischen 1970 und 1990 wuchs die Zahl der in größerem Umfang von Auswanderung betroffenen Länder von 29 auf 55 und die Zahl der gewichtigen Einwanderungsländer verdoppelte sich von von 39 auf 76 (Stalker 2000). Der „Export” von Arbeitskraft aus südostasiatischen Ländern wie Thailand, Indonesien und den Philippinen und aus den südasiatischen Staaten Indien, Bangladesh, Sri Lanka und Pakistan ist seit den 1980er Jahren um ein Vielfaches gestiegen. Von dem letztgenannten Land ist mittlerweile rund ein Fünftel der Erwerbsbevölkerung im Ausland tätig. Hier wie auch in einer Reihe anderer Staaten fördert die Regierung die Migration zur Minderung des Angebotsdrucks auf dem Arbeitsmarkt und als Einkommensquelle aus den Geldüberweisungen der im Ausland tätigen Arbeitskräfte. Für das Jahr 2005 wurde der globale Gesamtwert dieser Überweisungen von der Weltbank mit 232 Mrd. US \$ angegeben, wobei die größten Beträge auf Indien (22 Mrd.), China (21 Mrd.), Mexiko (18 Mrd.) und die Philippinen (12 Mrd.) entfallen. Für das letztgenannte Land machen die Überweisungen 13,5\% des Nationaleinkommens aus (vgl. BBC News, http://news.bbc.co.uk/go/pr/fr/-/2/hi/business/4608786.stm). Seit 1990 hat sich insbesondere der Zustrom qualifizierter und hochqualifizierter Arbeitskräfte aus den Entwicklungsländern in die entwickelte Welt (besonders USA, Kanada, Australien und Westeuropa) und in die arabischen Golfstaaten erheblich verstärkt. So sind beispielsweise Arbeitskräfte mit dem höchsten Bildungs- und Ausbildungsstand aus Mexiko, Guatemala, El Salvador, Jamaika und der Dominikanischen Republik in die USA ausgewandert (Adams 2003). Dies mindert die Entwicklungschancen des Südens und potenziert das Entwicklungsgefälle zum Norden, insbesondere dann, wenn die Migranten nicht in ihre Heimatländer zurückkehren.

\section{$4 \quad$ Zusammenfassung}

Die wirtschaftlichen und sozialen Wirkungen der weltwirtschaftlichen Integration während der letzten drei Jahrzehnte waren höchst uneinheitlich. Eine kleine Zahl von Staaten, vor allem in Ost- und Südostasien, hat von der Teilhabe am Globalisierungsprozess profitiert. Durch aktive, staatliche Steuerung der Marktöffnung konnten sie hohes, beschäftigungsintensives Wachstum und eine Reduzierung der Arbeitslosigkeit, Unterbeschäftigung und informeller Beschäftigung erzielen, die Realeinkommen anheben und das Ausmaß extremer Armut verringern.

Die Mehrzahl der Entwicklungsländer jedoch, insbesondere jene in Lateinamerika und südlich der Sahara, konnte durch die Globalisierung wenig gewinnen und fiel im Entwicklungsniveau gegenüber den Industriestaaten und den asiatischen Schwellenländern erheblich zurück. Gemessen am durchschnittlichen BIP pro Kopf erhöhte sich das Einkommensgefälle zwischen armen und reichen Staaten. Die Verheißung einer Angleichung der Arbeits- und 
Lebensverhältnisse mittels Liberalisierung der Märkte blieb weitgehend unerfüllt. Vielerorts war die Realeinkommensentwicklung negativ, informelle Beschäftigung und Armut wuchsen rapide. Nahezu kein Entwicklungsland, eingeschlossen die dynamischen asiatischen Schwellenländer, war von einer Zunahme relativer Armut, primär bedingt durch verstärkte Einkommenspreizung, verschont-erfahrungsgemäß ein Vorbote innerer sozialer Spannungen und politischer Instabilität. Da sich auch in nahezu allen Industriestaaten und den Ländern im Übergang von der Zentralverwaltungs- zur Marktwirtschaft verstärkte Ungleichheit der Einkommen herausgebildet hat, kann man von einer mehrdimensionalen globalen Spaltung sprechen: Arm und Reich driften innerhalb und zwischen den Nationen auseinander.

\section{Literatur}

Adams, Richard H. Jr. (2003): International Migration, Remittances, and the Brain Drain: A Study of 24 Labor-Exporting Countries. Policy Research Paper No. 3969 (May), World Bank, Washington, D.C.

Chen, Shaohua, Martin Ravallion (2005): How Have the World's Poorest fared since the Early 1980s?, Unpublished Paper, World Bank, Washington, D.C.

Cornia, Giovanni Andrea (2003): The Impact of Liberalization and Globalization on Income Inequality; in: Developing and Transitional Economies, Policy Integration Dept., ILO, Working Paper

Daza, José Luis (2005): Informal Economy, Undeclared Work and Labour Administration, Paper No. 9, Social Dialogue, Labour Law and Labour Administration Dept, International Labour Office, Geneva

Gunter, B.G., Ralph van der Hoeven (2004): The Social Dimension of Globalization: A Review of the Literature; in: International Labour Review, Vol. 143, No. 1-2, ILO, Geneva

ILO (2002a): Decent Work and the Informal Economy, Report IV, International Labour Conference, 90th Session 2002, Geneva

ILO (2002b): A Future Without Child Labour. Global Report Under the Follow-up of the ILO Declaration Fundamental Principles and Rights at Work, Geneva

ILO (2003a): Global Employment Trends, Geneva

ILO (2003b): Key Indicators of the Labour Market (KILM), 3rd Edition, Geneva

ILO (2005a): World Employment Report 2004-05: Employment, Productivity and Poverty Reduction, Geneva

ILO (2005b): A Global Alliance Against Forced Labour, Global Report under the Follow-up to the ILO Declaration on Fundamental Principles and Rights at Work, International Labour Conference, 93th Session 2005, Geneva

Kapsos, Steven (2004): Estimating Growth Requirements for Reducing Working Poverty: Can the World Halve Working Poverty by 2015? Employment Strategy Papers 2004/14, ILO, Geneva

Portes, Alejandro (1994): By-passing the Rules. The Dialectics of Labour Standards and Informalization in Less Developed Countries; in: Werner Sengenberger, Campbell (eds.); in: International Labour Standards and Economic Interdependence, IILS Geneva

Pries, Ludger (1992): Abhängige und selbständige Erwerbsarbeit in Lateinamerika: Eine empirische Überprüfung des Konzepts vom ‘Informellen Urbanen Sektor’; in: Kölner Zeitschrift für Soziologie und Sozialpsychologie, Jg. 44, 4, 655-676

Sengenberger, Werner (2005): The Role and Impact of International Labour Standards, Report for the Friedrich-Ebert-Foundation, Bonn (first edition 2002)

Sivananthiram, A., C. S. Venkata Ratnam (2005): Informal Economy: Growing Challenge for Labour Administration, ILO, Geneva

Stalker, Peter (2000): Workers Without Frontiers - The Impact of Globalization on International Migration, ILO, Geneva 
UNCTAD (2003): Trade and Development Report 2003, Geneva

UNDP (1999): Human Development Report, Geneva

UNDP (2002): Human Development Report, Geneva

Van Ginneken, Wouter (2003): Extending Social Security: Policies for Developing Countries; in: International Labour Review, ILO, Geneva, Vol. 142 (3), 277-294

World Bank (2005a): China and the WTO: Accession, Policy Reform and Poverty Reduction Strategies, Washington, D.C.

World Bank (2005b): Doing Business in 2006: Creating Jobs, Private Sector Department, World Bank, Washington, D.C.

World Commission on the Social Dimension of Globalization (2004): A Fair Globalization - Creating Opportunities for All. ILO, Geneva

Anschrift des Verfassers:

Prof. Dr. Werner Sengenberger

7 Les Collines de Pitegny

385 Chemin de I'Ovellas

F-01170 Gex, Frankreich

Email: Sengenberger.w@wanadoo.fr 